\title{
La cultura de paz como eje transversal en la formación de valores
}

The culture of peace as a transversal axis in the formation of values

\author{
Liliana Caterine Contreras-Garzón \\ lilika442@hotmail.com \\ Universidad Iberoamericana Internacional, Campeche \\ México \\ https://orcid.org/0000-0003-4608-5866 \\ María Ángeles Díaz-Linares \\ m.angeles.diaz.linares@hotmail.com \\ Universidad Iberoamericana Internacional, Campeche \\ México \\ https://orcid.org/0000-0003-3635-9528
}

Recepción: 10 de mayo 2021

Revisado: 15 de junio 2021

Aprobación: 15 de agosto 2021

Publicación: 01 de septiembre de 2021 


\title{
RESUMEN
}

La mediación que busca encontrar la igualdad y el respeto del otro, disminuyendo los desacuerdos entre los pares mantendrá un ambiente de paz desde la dinámica y promoción de los valores. En la siguiente investigación se planteó como objetivo general analizar la cultura de paz como eje transversal en la formación de valores en los niños y jóvenes estudiantes. Desde el enfoque cuantitativo, a través de la exploración, recolección y análisis crítico mediante una tipología documental, con un diseño bibliográfico de tipo documental, que busca la reflexión y análisis. Se revisaron y analizaron fuentes documentales a nivel teórico y trabajos arbitrados vinculadas con los aspectos centrales del trabajo. La cultura de paz es promotora de la ética, desde la transversalidad. Se concluye que viene a romper los viejos esquemas didácticos, de instrucción, de enseñanza-aprendizaje por una nueva forma de mirar al otro, y contribuir al logro del bien de los ciudadanos.

Descriptores: Cultura de paz; sistema de valores; educación; formación; solución de conflictos. (Palabras tomadas del Tesauro UNESCO).

\begin{abstract}
Mediation that seeks to find equality and respect for the other, reducing disagreements between peers, will maintain an environment of peace from the dynamics and promotion of values. In the following research, the general objective was to analyze the culture of peace as a transversal axis in the formation of values in children and young students. From the quantitative approach, through exploration, collection and critical analysis through a documentary typology, with a bibliographic design of a documentary type, which seeks reflection and analysis. Documentary sources at a theoretical level and refereed works related to the central aspects of the work were reviewed and analyzed. The culture of peace is a promoter of ethics, from transversality. It is concluded that it comes to break the old didactic, instructional, teaching-learning schemes by a new way of looking at the other, and contributing to the achievement of the good of the citizens.
\end{abstract}

Descriptors: Peace culture; Values system; education; training; conflict resolution. (Words taken from the UNESCO Thesaurus). 


\section{INTRODUCCIÓN}

El interés para desarrollar la presente investigación surge a partir de la necesidad de fomentar la cultura de paz como eje transversal en la formación de valores en la educación primaria y secundaria en el currículo. Esto involucra, fortalecer los procesos de enseñanza aprendizaje desde una tarea innovadora propia del quehacer educativo, encaminadas a mejorar la comprensión y solución de los problemas y conflictos propios del contexto, teniendo en cuenta la percepción de la transversalidad de los valores en el campo pedagógico de los docentes con el objeto de la promoción de la paz. En este sentido, es de suma importancia indicar que la implementación de la cultura de paz como eje transversal en la formación de valores busca ser un modelo participativo, que plantea desarrollar en las comunidades educativas en general, valores y actitudes que favorecen la formación para la paz. La implementación y la justicia transicional, es la mirada territorial de las dinámicas del conflicto, los efectos de la violencia y la construcción de la paz. (Corcione Nieto, Fernández Osorio, Cabrera Cabrera,2021, p.12).

Hay que destacar, lamentablemente que en Colombia los hechos de violencia y la vulneración de la paz es de larga data, que se manifiesta en ataques armados, guerrilla, para militares, manifestaciones violentas, entre otras. Al respecto Quintero López (2020) manifiesta:

Actualmente en la sociedad persisten problemas de violencia, crímenes, discriminación, que opacan la sana convivencia de los individuos en los diversos estratos de la población, por lo que se hace necesario la promoción de una Cultura de Paz en la que se priorice el respeto y tolerancia hacia los demás (p.124)

Por tal razón, para entender la problemática de la vulneración de la paz es significativo apoyarse en otras ciencias y no circunscribirse a una sola área del conocimiento, ya que el encuentro y la contribución de diferentes disciplinas formularán estrategias de perfeccionamiento fundamentadas en mejorar la situación problemática. Así mismo, a nivel del Estado colombiano en sus políticas publicas plantean, según Delgado Barón 
EPISTEME KOINONIA
Revista Electrónica de Ciencias de la Educación, Humanidades, Artes y Bellas Artes Año IV. Vol IV. N ${ }^{\circ}$. Julio - Diciembre. 2021
Hecho el depósito de Ley: FA2018000022 ISSN: 2665-0282

(2011) la siguiente ley:

Con la Ley de Justicia y Paz el gobierno colombiano busca dar un sustento jurídico al proceso de desmantelamiento de las estructuras de los grupos armados al margen de la ley, haciendo un especial énfasis en el carácter de reconciliación que implican las desmovilizaciones, como un mecanismo adoptado para contribuir a la "consecución de la paz". De este modo, la Ley estipula que dentro del proceso de reconciliación nacional es necesario el derecho a la verdad, la justicia y la reparación para las víctimas. (p.182)

Intensificar la cultura de paz como eje transversal en la formación de valores en las escuelas y por ende en los planes de estudios, involucra el fortalecimiento los procesos desde una faena innovadora, además es importante el apoyo del personal docente, con el deseo de brindar lo mejor de sí, al respecto del Salto Bello (2015) manifiesta:

Para la formación en valores se necesita una educación donde el profesorado adquiera un compromiso social y ético, que colabore con las alumnas y los alumnos para facilitarles la creación y el desarrollo de capacidades que intervengan en el juicio y la acción moral, de manera que se posibilite la formación de actitudes, la integración, la aplicación y la valoración crítica de las normas que rigen en una sociedad. (p.1422)

De este modo, los planes de estudio, los currículos, los procedimientos pedagógicos en las instituciones de educación, están llamadas a estructurarse y establecer una interrelación y coordinación con las diferentes áreas con el objeto de enfrentar una problemática. Por ello, el impulso humano sostenible y la problemática en la ausencia de valores puede ser abordado dese varias disciplinas, en la que respondan a los principales problemas, con los aportes de los docentes desde las diferentes disciplinas, ofreciendo una visión desde su especialidad. El esfuerzo generalizado para modificar mentalidades y actitudes con ánimo de promover la paz. Significa transformar los conflictos, prevenir los que puedan engendrar violencia y restaurar la paz y la confianza en poblaciones que emergen de la guerra (Rojas Bonilla, 2018, p.2). 
En este sentido, Ochoa Rocha (2021) manifiesta:

Las instituciones educativas en cualquiera de sus niveles están obligadas a formar sujetos integrales con conocimientos científicos de las diferentes áreas de la ciencia y la tecnología, pero a su vez también se requiere que se vean interesados y formados en adquirir valores morales, democráticos y de respeto a los Derechos Humanos de todas las personas, con el compromiso de velar por un desarrollo sustentable, con la convicción de buscar la igualdad entre hombres y mujeres, con el libre ejercicio de atender a las problemáticas sociales y enmarcados en la resolución pacífica de conflictos y la sana convivencia.(p.95)

El sistema educativo demanda conocer la naturaleza de los conflictos para generar posibilidades de paz vinculada a los derechos humanos, se debe adaptar de manera cotidiana en la que los seres humanos se relacionan entre sí. Para cristianizar en una gran vía que favorezca la cultura de paz estableciendo la misión educativa en todos sus niveles desde la formación inicial hasta la universitaria en medio de la perturbación que se vive día a día en la cotidianidad y realidad específica de cada ser que se ve sensible ante los diferentes problemas. En este período el país se encuentra ensimismada en grandes conflictos producto de desacuerdos compartidos perturbando todos los niveles y estratos sociales. En este análisis reflexivo la palabra de Martínez Real (2012) hacen eco en el quehacer educativo:

Reflexionemos entonces sobre nuestra función como docentes. ¿Tendremos los maestros y las maestras responsabilidad de apoyar, guiar a los alumnos para que logren una formación que les permita convertir en habilidades las potencialidades que como seres humanos tienen? ¿Debemos los maestros y las maestras enseñarlos a ser mejores seres humanos? Consideramos que sí, y es por esta perspectiva moral y ética que afirmamos que uno de los principales compromisos de los y las docentes es trabajar con la Educación para la paz (p.73)

El proyecto de la paz en el tejido educativo como eje transversal en la formación de valores, en el significado y sentido de las ideologías de la paz en el conjunto de elementos, factores y ambientes educativos, favorece a los alumnos, estudiantes, docentes, maestros y participantes habituarse, con las contradicciones de interes442es 
como exigencias para generar y asimilar discernimientos que justifiquen la programación y apropiarse de la responsabilidad de los recursos manejados en el proceso de enseñanza-aprendizaje como medio del trabajo preventivo asistiendo las acciones conjuntas de las capacidades-aptitudes de los individuos y sus relaciones alcanzando los cimientos para la gestión del aprendizaje. Esta forma de deliberar desde la paz en el contexto educativo debe reorientar las formas de coexistencias en todos los ambientes en donde se desenvuelven los actores de la comunidad educativa como una correlación alumno-docente-padres-comunidad. El contexto escolar emerge como escenario donde se ven reflejadas las dinámicas sociales, tanto del conflicto como de los propósitos por construir una cultura de paz, orientadas desde los órganos de gobierno que mediar en la convivencia escolar. (Cardozo Rusinque, Morales Cuadro y Martínez Sande, 2020, p.3). Al respecto, es necesario la toma de conciencia de acuerdo al planteamiento de Romero Ramírez (2020):

Las difíciles circunstancias que rodean los centros educativos: violencia, falta de respeto e indisciplina, falta de recursos, desencuentro con los padres, ha desencadenado una verdadera crisis de valores donde la autoridad es desconocida, la persona es asumida en muchos casos como objeto de placer sin importar los riegos que se tomen, la pereza, se alza frente a las ganas de hacer, de construir, la inmediatez de las cosas se impone y el conocimiento es visto como un elemento poco o nada atractivo, lo que lleva a restar no sólo al estudiante en su ser persona, sino también la labor docente.(30)

En este sentido romper con la mala imagen de que los enfrentamientos, las guerras y la violencia es normal e inevitable y es la elección frente a los conflictos es significativo la educación como estrategia y herramienta de evolución pacifica de los conflictos humanos. "Se trata de una educación con amor que nos permite mejorar nuestro mundo y disfrutar de nuestras experiencias con el otro diferente, sin prejuicios ni discriminaciones. (Bahajin, 2018, p.95). Así mismo, Echeverri Álvarez, Giraldo Ramírez y Restrepo Mesa (2020) manifiestan que: 
La paz no es un regalo que viene de afuera, sino una disciplina de construcción, diariamente, por la misma comunidad. Por eso, entonces, se concluye que para que la educación pueda construir una cultura de paz, primero tiene que ser capaz de ver la propia cultura y, con imaginación pedagógica, ponerla al servicio del alcance de fines específicos, como la paz, por ejemplo. (p.786)

Instruir para la paz envuelve educar sobre los conflictos, las discrepancias, disconformidades, las injusticias, el quebrantamiento de los valores entre otros. La educación favorece a la formación de ciudadanos, pueblos y naciones con el soporte de las variadas herramientas y métodos pedagógicos de enseñanza-aprendizaje para el desarrollo cognitivo. En este, sentido Vidal Ledo y Araña Pérez (2014) plantean la importancia de una formación en valores:

La necesidad de profundizar y fortalecer la educación en valores por todos los factores de la sociedad, es una tarea importante hoy más que nunca en todo el mundo. De ello depende salvar a lo más preciado que ha existido en nuestro planeta: el ser humano. (p.183)

Por todo lo desarrollado se plantea como objetivo de la investigación analizar la cultura de paz como eje transversal en la formación de valores en los niños y jóvenes estudiantes. escolar.

\section{METODOLOGÍA}

El trabajo de investigación se recorrió desde el enfoque cuantitativo, a través de la exploración, recolección y análisis crítico mediante una tipología documental, con un diseño bibliográfico de tipo documental, que busca la reflexión y análisis construyendo métodos relacionados al discernimiento del fenómeno y así evaluar o considerar nuevos contextos. Al respecto, Palella y Martins (2012) manifiestan, la investigación documental se encuentra exclusivamente en la recolección de información en diversas fuentes. Adicionalmente, se revisaron y analizaron fuentes documentales a nivel teórico y trabajos arbitrados vinculadas con los aspectos centrales del trabajo: la cultura de paz como eje 
trasversal en la formación de valores, especialmente en las instituciones de educación.

\section{RESULTADOS}

En cuanto a los resultados se presenta una recopilación de trabajos científicos y arbitrados que abordan el tema investigado como corpus teórico que contribuye al fortalecimiento de la temática desarrollada.

\section{Cuadro 1.}

Visión conceptual.

\begin{tabular}{|c|c|c|}
\hline Autor & Titulo & Planteamiento \\
\hline $\begin{array}{l}\text { Álvarez-Maestre, A. J. y } \\
\text { Pérez-Fuentes, C. A. } \\
\text { (2019). }\end{array}$ & $\begin{array}{l}\text { Educación para la paz: } \\
\text { aproximación teórica desde } \\
\text { los imaginarios de paz. }\end{array}$ & $\begin{array}{l}\text { El giro epistemológico } \\
\text { propuesto por Galtung } \\
\text { propone: "si quieres la paz, } \\
\text { prepárate para la paz", en } \\
\text { contraposición al dictum "si } \\
\text { quieres la paz, prepárate } \\
\text { para la guerra", con lo que } \\
\text { se abre la posibilidad para } \\
\text { la humanidad de observar } \\
\text { una antropología que confía } \\
\text { en el hombre, en los } \\
\text { mecanismos de resolución } \\
\text { pacífica de conflictos, en la } \\
\text { construcción de la paz y en } \\
\text { el fortalecimiento de la } \\
\text { ética. (p281). }\end{array}$ \\
\hline $\begin{array}{l}\text { Miló León, y Valle Gálvez } \\
\text { (2019) }\end{array}$ & $\begin{array}{l}\text { La educación para la paz } \\
\text { como competencia para la } \\
\text { vida: una mirada desde la } \\
\text { labor del docente. }\end{array}$ & $\begin{array}{l}\text { La educación para la paz } \\
\text { ofrece el marco idóneo en la } \\
\text { formación de este } \\
\text { profesional para trabajar } \\
\text { con herramientas que } \\
\text { contribuyan desde los } \\
\text { componentes académico, } \\
\text { laboral, investigativo y } \\
\text { extensionista en la solución } \\
\text { de problemas } \\
\text { profesionales, en vínculo }\end{array}$ \\
\hline
\end{tabular}




\begin{tabular}{|c|c|c|}
\hline & & $\begin{array}{l}\text { estrecho con la solución } \\
\text { pacífica del conflicto, } \\
\text { promoviendo la adquisición } \\
\text { de valores que contribuyan } \\
\text { al desarrollo integral de este } \\
\text { profesional como ser } \\
\text { humano. (p.3). }\end{array}$ \\
\hline $\begin{array}{l}\text { Esquivel Marín, C., y } \\
\text { García Barrera, M. (2018) }\end{array}$ & $\begin{array}{l}\text { La Educación para la Paz y } \\
\text { los Derechos Humanos en } \\
\text { la creación de valores para } \\
\text { la solución de conflictos } \\
\text { escolares. }\end{array}$ & 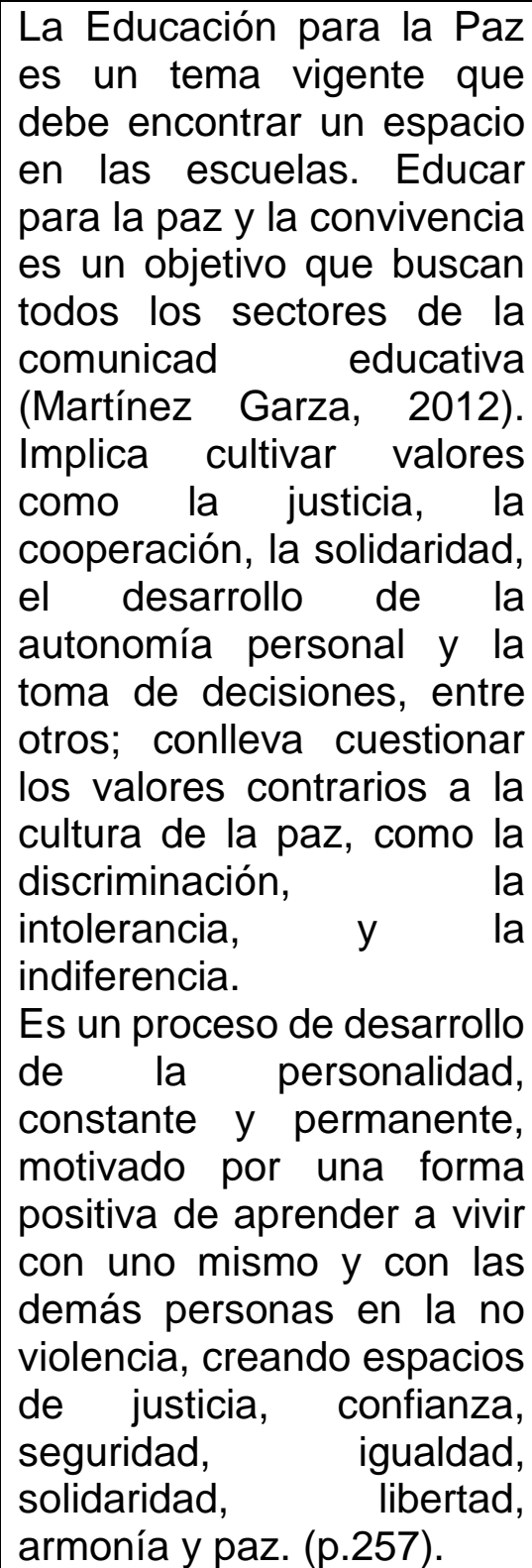 \\
\hline Archundia & Conflicto & Ofrecer \\
\hline
\end{tabular}




\begin{tabular}{|c|c|c|}
\hline Gutiérrez Méndez (2016) & Instituciones Escolares. & $\begin{array}{l}\text { integrado desde la visión } \\
\text { conflictiva de la realidad y } \\
\text { en el que se cuestione la } \\
\text { violencia como forma de } \\
\text { transformación de los } \\
\text { conflictos. Por } \\
\text { consiguiente, no se trata de } \\
\text { negar las diferencias y los } \\
\text { conflictos sino de } \\
\text { afrontarlos de forma } \\
\text { positiva, es decir, de forma } \\
\text { no violenta. Afrontar los } \\
\text { desacuerdos no implica } \\
\text { generar dinámicas de } \\
\text { destrucción ni, en el otro } \\
\text { extremo, acomodarnos o } \\
\text { someternos a los } \\
\text { requerimientos de la otra } \\
\text { parte. (p.172). }\end{array}$ \\
\hline $\begin{array}{l}\text { Chávez Romo, Ramos } \\
\text { Sánchez y Velázquez } \\
\text { Jaramillo (2017) }\end{array}$ & $\begin{array}{l}\text { Análisis de las estrategias } \\
\text { docentes para promover la } \\
\text { convivencia y disciplina en } \\
\text { el nivel de educación } \\
\text { preescolar. }\end{array}$ & $\begin{array}{l}\text { En este contexto cobra } \\
\text { relevancia que el sistema } \\
\text { educativo promueva, en los } \\
\text { niveles de educación } \\
\text { básica, experiencias } \\
\text { significativas donde los } \\
\text { niños tengan la oportunidad } \\
\text { de reconocerse personas } \\
\text { valiosas, dignas de un trato } \\
\text { justo y respetuoso, capaces } \\
\text { de gozar sus libertades } \\
\text { fundamentales y ejercerlas } \\
\text { responsablemente, así } \\
\text { como ensayar formas de } \\
\text { resolver los conflictos } \\
\text { interpersonales que surgen } \\
\text { en el contacto con sus } \\
\text { pares. (p.60) }\end{array}$ \\
\hline Catzoli Robles (2016) & $\begin{array}{l}\text { Concepción de paz y } \\
\text { convivencia en el Contexto } \\
\text { escolar }\end{array}$ & $\begin{array}{l}\text { La educación para la paz no } \\
\text { es una opción más sino una } \\
\text { necesidad que toda } \\
\text { institución educativa debe }\end{array}$ \\
\hline
\end{tabular}




\begin{tabular}{|c|c|c|}
\hline & & $\begin{array}{l}\text { asumir. Los principios para } \\
\text { una convivencia pacífica } \\
\text { entre pueblos y grupos } \\
\text { sociales se han convertido } \\
\text { en un imperativo legal. } \\
\text { Ahora se trata de conseguir } \\
\text { que el derecho formal de la } \\
\text { paz se convierta en un } \\
\text { derecho real.(p.435) }\end{array}$ \\
\hline $\begin{array}{lcc}\text { González } & \text { Rodríguez y } \\
\text { Cardentey García (2016) }\end{array}$ & $\begin{array}{l}\text { Educación en valores de } \\
\text { estudiantes universitarios }\end{array}$ & $\begin{array}{l}\text { El proceso } \\
\text { educativo está concebido } \\
\text { para educar valores, } \\
\text { particularmente } \\
\text { estudiantes universitarios; } \\
\text { para lograrlo se necesita } \\
\text { encaminar el mismo hacia } \\
\text { el modelo ideal de } \\
\text { formación, desarrollar el } \\
\text { vínculo con la realidad a } \\
\text { través de la formación socio } \\
\text { humanista y determinar } \\
\text { estrategias didácticas que } \\
\text { involucren a los galenos en } \\
\text { una actividad consciente, } \\
\text { protagónica } \\
\text { comprometida. (164) y }\end{array}$ \\
\hline Rosendo López (2020) & $\begin{array}{l}\text { Valores En La Cultura De } \\
\text { Paz: Camino Para La } \\
\text { Prevención De Violencia } \\
\text { Escolar. }\end{array}$ & $\begin{array}{l}\text { La educación en valores } \\
\text { juega un papel } \\
\text { predominante en la } \\
\text { construcción de la cultura } \\
\text { de paz no solo en la } \\
\text { escuela, sino también en la } \\
\text { sociedad en general, por } \\
\text { cuanto si educamos a los } \\
\text { niños, niñas, jóvenes y } \\
\text { adolescentes con respecto } \\
\text { a los valores humanos } \\
\text { entonces se tendrán } \\
\text { mejores estudiantes y } \\
\text { ciudadanos que coadyuven } \\
\text { a mejorar la convivencia en }\end{array}$ \\
\hline
\end{tabular}




\begin{tabular}{|l|l|l|}
\hline & & $\begin{array}{l}\text { las escuelas y y } \\
\text { comunidades en las cuales } \\
\text { viven. (41) }\end{array}$ \\
\hline Silva (2015) & $\begin{array}{l}\text { Educar en los valores } \\
\text { universales de la cultura de } \\
\text { paz }\end{array}$ & $\begin{array}{l}\text { Lo fundamental de la } \\
\text { educación para la paz es } \\
\text { que su mismo concepto es } \\
\text { una herramienta para la } \\
\text { actividad, los medios y el fin } \\
\text { de esta educación } \\
\text { comprensiva como parte } \\
\text { del conjunto de la } \\
\text { educación en valores. } \\
\text { (p.29) }\end{array}$ \\
\hline
\end{tabular}

Elaboración: Los autores.

Sin duda alguna la cultura de paz como eje transversal en la formación de valores, para el campo educativo es de suma importancia y relevancia para complementar los procesos de enseñanza aprendizaje para la formación integral de los ciudadanos y futuros profesionales. Lo fundamental de la educación para la paz es que su mismo concepto es una herramienta para la actividad, los medios y el fin de esta educación comprensiva como parte del conjunto de la educación en valores. (Silva, 2015, p.29). Es convocar a la búsqueda de afianzar los valores para una vida plena, es decir, hacer de la paz un proyecto de vida implica que hay una responsabilidad social, ética, pedagógica, cultural y política frente a las posibilidades de autogestión de la vida. (Oliveros Ossa, Palacios Romaña y Correa Villa, 2021, p.3)

Por lo tanto, la convivencia en los diferentes escenarios del vivir diario, deberá estar cimentado en el respeto a las diferencias que se generan constantemente, al respecto Pirona (2018) plantea:

El comportamiento humano es entendido, desde la ética, como una tarea de construcción personal, de desarrollo creativo de una vida armónica con uno mismo y con el entorno, de la búsqueda de la excelencia, de la mejora continua, que requiere un renovado esfuerzo (p.50) 
Siguiendo esta línea, la cultura de paz es promotora de la ética, desde la transversalidad ya que promueve los valores del respeto, de la vida, la convivencia, la cooperación. Es así como Rodríguez (2016) manifiesta:

La imperiosa obligación de educar para la paz, como el pilar fundamental, como la respuesta a tantas carencias, pero sobre todo como la posibilidad de generar profundos cambios en la estructura familiar, social, ideológica y educativa y educar para la paz debe partir necesariamente de una educación en valores (p.7)

\section{CONCLUSIONES}

En los tiempos actuales en el que continuamente se producen cambios en lo social, político, económico, salud, ambiental, tecnológicos, educativos entre otros, las instituciones educativas y universitarias, están emplazadas a formalizar trasformaciones e innovaciones en los contenidos de los planes, programas y estrategias pedagógicos; estos cambios fundamentales han de estar integrados en todas las unidades académicas, áreas y materias que se ofrecen en las instituciones educativas en todos los niveles de formación desarrollando, repensando, promocionando nuevos paradigmas que promuevan al nuevo ciudadano considerando los requerimientos y necesidades del entorno para dar respuestas a los desacuerdo y divergencia que se manejan desde el plano de la violencia y vulneración de los derechos ciudadanos.

Por ello, la cultura de paz como eje trasversal en la formación de valores, viene a romper los viejos esquemas didácticos, de instrucción, de enseñanza-aprendizaje, impositivo, egoísta, y centrista por una nueva forma de mirar al otro, y contribuir al logro del bien de los ciudadanos.

\section{FINANCIAMIENTO}

No monetario. 


\section{AGRADECIMIENTO}

A la Universidad Iberoamericana Internacional; por motivar el desarrollo de la investigación.

\section{REFERENCIAS CONSULTADAS}

Álvarez-Maestre, A. J. y Pérez-Fuentes, C. A. (2019). Educación para la paz: aproximación teórica desde los imaginarios de paz. [Education for peace: theoretical approach from the imaginaries of peace]. Educación y Educadores, 22(2), 277-296. DOI: https://doi.org/10.5294/edu.2019.22.2.6

Bahajin, S. (2018). La educación como instrumento de la cultura de paz. [Education as an instrument of the culture of peace]. Innovación educativa (México, DF), 18(78), 93-111. Recuperado de: https://n9.cl/s3paw

Cardozo Rusinque, A., Morales Cuadro, A., y Martínez Sande, P. (2020). Construcción de paz y ciudadanía en la Educación Secundaria y Media. En Colombia. [Peacebuilding and citizenship in Secondary and Middle Education. In Colombia] Educação e Pesquisa. 46: DOI: http://dx.doi.org/10.1590/S1678$\underline{4634202046214753}$

Corcione Nieto, M., Fernández Osorio, A. y Cabrera Cabrera, L. (2021) Academia, conflicto armado y paz en Colombia: Un Acercamiento desde la Geopolítica del Conocimiento. [Academia, armed conflict and peace in Colombia: An Approach from the Geopolitics of Knowledge]. DADOS. 64(4), 1-46. https://doi.org/10.1590/dados.2021.64.4.247

Catzoli Robles, Lucero (2016). Concepción de paz y convivencia en el contexto escolar. [Conception of peace and coexistence in the school context]. Ra Ximhai, 12(3),433-444. Recuperado de: https://n9.cl/0b6dn

Chávez Romo, M., Ramos Sánchez, A., y Velázquez Jaramillo, P. (2017). Análisis de las estrategias docentes para promover la convivencia y disciplina en el nivel de educación preescolar. [Analysis of teaching strategies to promote coexistence and discipline at the preschool education level]. Educación, 26(51),3554. https://dx.doi.org/10.18800/educacion.201702.003 
Delgado Barón, M. (2011). La Ley de Justicia y Paz en Colombia: La configuración de un subcampo jurídico-político y las luchas simbólicas por la inclusión. [The configuration of a legal-political subfield and the symbolic struggles for inclusion]. Revista de Relaciones Internacionales, Estrategia y Seguridad, 6(2), 179-194. Recuperado de: https://n9.cl/3szjk

del Salto Bello, M. (2015). Educación en valores: propuesta de una estrategia. [Education in values: proposing a strategy]. MEDISAN, 19(11), 1421-1429. Recuperado de: https://n9.cl/phoh8

Echeverri-Álvarez, J., Giraldo-Ramírez, M. y Restrepo Mesa, K. (2020) Creando Paz: Contenidos formativos con imaginación pedagógica. [Creating Peace: Training Contents with Pedagogical Imagination]. Cad. Pesqui., 50(177),774-789. https://doi.org/10.1590/198053146935

Esquivel Marín, C., y García Barrera, M. (2018). La Educación para la paz y los derechos humanos en la creación de valores para la solución de conflictos escolares. [Education for peace and human rights in the creation of values for the resolution of school conflicts]. Revista Justicia, 23(33), 256-270. https://doi.org/10.17081/just.23.33.2892

González Rodríguez, R., y Cardentey García, J. (2016). Educación en valores de estudiantes universitarios. [Education in values of university students]. Humanidades Médicas, 16(1), 161-174. Recuperado de: https://n9.cl/gl27e

Martínez Real, C. (2012) La educación para la paz como eje transversal en el nivel medio superior. [Peace education as a transversal axis at the upper secondary level]. Ra Ximhai, 8(2), 71-91. Universidad Autónoma Indígena de México El Fuerte, México. Recuperado de: https://n9.cl/01815

Miló León, O., Breijo Worosz, T. y Valle Gálvez, C. (2019). La educación para la paz como competencia para la vida: una mirada desde la labor del docente. [Education for peace as a competition for life: a look from the work of the teacher]. Varona. Revista Científico Metodológica, (69),1-4. Recuperado de: https://n9.cl/b38r2 
Ochoa Rocha, J. (2021). La transversalidad de la cultura de paz en la educación superior como eje principal para la formación integral de profesionistas. [The transversality of the culture of peace in higher education as the main axis for the integral formation of professionals]. Eirene Estudios de Paz y Conflictos, 4(6), 93-112. Recuperado de: https://n9.cl/p56lh

Oliveros Ossa, J., Palacios Romaña, E., y Correa Villa, C. (2021). Paz es entender lo que somos: prácticas socioculturales de paz en Quibdó. [Peace is understanding what we are: socio-cultural practices of peace in Quibdó]. RHS-Revista Humanismo $Y$ Sociedad, 9(1), 1 - 16. https://doi.org/10.22209/rhs.v9n1a01

Palella, S. y Martins, F. (2012). Metodología de la Investigación Cuantitativa. [Quantitative Research Methodology]. Fondo Editorial de la Universidad Pedagógica Experimental Libertador. Caracas. Venezuela

Pérez Archundia, E. y Gutiérrez Méndez, D. (2016). El Conflicto en las Instituciones Escolares. [ Conflict in School Institutions]. Ra Ximhai,12, (3), 163-180. Recuperado de: $\underline{\text { https://n9.cl/ihn4b }}$

Pirona, J. (2018). Ética en la gestión universitaria perspectiva del pensamiento complejo. [Ethics in university management perspective of complex thinking]. Tesis Doctoral en Ciencias Gerenciales. UNEFA. Venezuela

Quintero López, I. (2020). Gestión de conflictos y mediación escolar en alumnos de la licenciatura en ciencias de la educación como herramientas para el desarrollo de una cultura de la paz. [Conflict management and school mediation in students of the bachelor's degree in education sciences as tools for the development of a culture of peace]. Conrado, 16(72), 123-130. Recuperado de: https://n9.cl/muk06

Rodríguez, N. (2016) Reflexión pedagógica educar para la paz: Una postura ética en el contexto educativo contemporáneo. [Pedagogical Reflection Educating for Peace: An Ethical Stance in the Contemporary Educational Contex]. Universidad Militar Nueva Granada. Recuperado de: https://n9.cl/a6vzu

Rojas Bonilla, E. (2018). La cultura de paz y su importancia en el proceso de formación ciudadana en el contexto educativo colombiano. [The culture of peace and its importance in the process of citizen formation in the Colombian educational context]. Varona. Revista Científico Metodológica, 66(1),1-4. Recuperado de: https://n9.cl/xa326 
Romero Ramírez, M. (2020). Vocación docente como respuesta esperanzadora en contextos de vínculos humanos frágiles. [Teaching vocation as a hopeful response in contexts of fragile human ties]. EPISTEME KOINONIA,3(5),27-50. http://dx.doi.org/10.35381/e.k.v3i5.527

Rosendo López. (2020) Valores en la cultura de paz: Camino para la prevención de violencia escolar. [Values in the peace culture: Way to prevent school violence]. Prohominum. Rev. de Ciencias Sociales y humanas, 2(4), 30-50. DOI: https://doi.org/10.47606/ACVEN/PH0017

Silva, E. (2015). Educar en los valores universales de la cultura de paz. [Educate in the universal values of the culture of peace]. Cultura De Paz, 21(66), 16-31. https://doi.org/10.5377/cultura.v21i66.2212

Vidal Ledo, M. y Araña Pérez, A. (2014) Formación en valores. [Formation of values]. Educación Médica Superior. 28(1):175-186. Recuperado de: https://n9.cl/6rnbc 